\title{
Proposta de um protocolo para reabilitação vestibular em vestibulopatias periféricas
}

\author{
Protocol's proposal for vestibular rehabilitation in outlying vestibulopatia
}

\author{
Adriana Roberta Degressi Rogatto ${ }^{[a]}$, Laira Pedroso ${ }^{[\mathrm{b}]}$, Sara Regina Meira Almeida ${ }^{[\mathrm{c}]}$, \\ Telma Dagmar Oberg ${ }^{[\mathrm{d}]}$
}

[a] Fisioterapeuta, Especialista em Fisioterapia Aplicada à Neurologia Adulto da Escola de Extensão da Universidade Estadual de Campinas (Extecamp-Unicamp), Campinas, SP - Brasil, e-mail: ardegressi@ig.com.br

[b] Fisioterapeuta, Especialista em Fisioterapia Aplicada à Neurologia Adulto, da Escola de Extensão da Universidade Estadual de Campinas (Extecamp-Unicamp), Hortolândia, SP - Brasil, e-mail: laira.pedroso@gmail.com

[c] Fisioterapeuta, Mestre em Ciências Médicas, Neurologia, Faculdade de Ciências Médicas da Universidade Estadual de Campinas (Unicamp), Campinas, SP - Brasil, e-mail: sararm@fcm.unicamp.br

[d] Fisioterapeuta, Doutora em Ciências Médicas, Neurologia, Faculdade de Ciências Médicas da Universidade Estadual de Campinas (Unicamp), Campinas, SP - Brasil, e-mail: oberg@fcm.unicamp.br

\section{Resumo}

Introdução: A reabilitação vestibular tem sido reconhecida como tratamento de escolha para pacientes com persistência da vertigem, por causa da disfunção vestibular periférica, proporcionando acentuada melhora na qualidade de vida. Dentre os tratamentos indicados, tem-se os exercícios de Cawthorne e Cooksey, integração sensorial e plataformas com movimentação corpórea. Objetivo: O objetivo da pesquisa foi criar um protocolo de exercícios em um balanço, associando os exercícios de Cawthorne e Cooksey à Integração Sensorial. Metodologia: Foi desenvolvido um relato de caso para avaliar uma paciente por meio das seguintes escalas: Escala de Equilíbrio de Berg e Dynamic Gait Índex (DGI) para verificar o equilíbrio; Five Times Sit-to-Stand (FTSTS) para avaliar a atividade de sentar e levantar; Dizziness Handicap Inventory (DHI) para verificar sintomas de vertigem; Escala de Sintomas após Tratamento Fisioterapêutico (ESATF), que gradua a sintomatologia após exercícios. Foi desenvolvido um protocolo associando as duas técnicas, nas quais os exercícios oculares, cefálicos e de tronco eram associados ao balanço. Foram realizadas 10 das 20 sessões previstas, por causa da frequência irregular do paciente às sessões. Resultados: O paciente melhorou de 19 para 22 pontos na DGI, manteve a pontuação na Berg e melhorou de 74 para 67 pontos na DHI. Na escala FTSTS, diminuiu o tempo de 15 para 14 segundos para realizar atividade de sentar e levantar. Na ESATF, houve oscilação da pontuação. Conclusões: Após o tratamento, a paciente manteve e obteve melhora no equilíbrio e na capacidade funcional e diminuiu o tempo gasto para realizar a atividade de sentar e levantar cinco vezes de uma cadeira com os membros superiores cruzados. Estudos adicionais com o protocolo proposto devem ser realizados, com um número maior de pacientes e maior assiduidade para haver habituação e reabilitação mais precoce.

Palavras-chave: Equilíbrio. Exercícios. Reabilitação vestibular. Cawthorne e Cooksey. Integração sensorial. 


\begin{abstract}
Introduction: Vestibular rehabilitation has been recognized as a treatment of choice for patients with persistent vertigo due to peripheral vestibular dysfunction, providing significant improvements in quality of life. Among the treatments listed have been exercises Cawthorne and Cooksey, sensory integration and body-moving platforms. Objective: The research objective was to invent the protocol of exercises in balance with the exercises of Cawthorne and Cooksey associated to the Sensory Integrative. Method: It was developed a pilot case, where was evaluated in the patient the balance for the Scale of Balance of Berg and Dynamic Gait Index (DGI); Five Times Sit-to-Stand (FTSTS), that evaluate the activity to sit down and to get up; Dizziness Handicap Inventory (DHI) that evaluate symptoms of vertigo; Scale of Symptoms after Physiotherapeutic Treatment (ESATF), graduates the symptoms after exercises. It was developed a protocol associating 2 techniques, which the ocular exercises, cephalic and of trunk were associated to the swinging. 10 of 20 sessions were accomplished, due to the patient's little regular attendance. Results: Patient obtained improvement from 19 to 22 in DGI; maintained the points of the Berg; she improved from 74 to 67 in DHI. In scale FTSTS she decreased the time from 15 to 14 seconds to accomplish activities of to sit down and to get up. In ESATF it happened oscillate in punctuation. Conclusion: After the treatment, the patient obtained an improvement in the appraised balance and improvement of the functional capacity, and she reduced the time spend to accomplish the activity of to sit down and to get up five times of a chair with the crossed arms. Additional studies with the proposed protocol should be accomplished, with a larger number of patients and regular attendance to have a faster adaptation.
\end{abstract}

Keywords: Balance. Exercises. Vestibular rehabilitation. Cawthorne e Cooksey. Sensory integrative.

\title{
Introdução
}

O sistema vestibular é responsável pela manutenção do equilíbrio geral, tem a função de informar sobre as acelerações angulares da cabeça nos diversos planos do espaço e movimentos corpóreos lineares (1). Quando sua função é interrompida, resultam sensações desagradáveis como: náuseas, vertigens, desequilíbrio e nistagmo (2).

Além do tratamento medicamentoso e cirúrgico, a reabilitação vestibular tem sido reconhecida como tratamento de escolha para pacientes com persistência da vertigem por causa da disfunção vestibular, proporcionando acentuada melhora na qualidade de vida (3).

Os objetivos principais da reabilitação vestibular são: promover a estabilização visual e aumentar a interação vestibulo-visual durante a movimentação da cabeça; proporcionar melhor estabilidade estática e dinâmica nas situações de conflito sensorial e diminuir a sensibilidade individual durante a movimentação cefálica (4).

Ganança e Caovilla (5) preconizam o programa de reabilitação vestibular em três elementos: exercícios de habituação que favorecem a compensação do sistema nervoso central, diminuindo ou abolindo as respostas anormais dos movimentos da cabeça, exercícios de controle postural e condicionamento das atividades gerais.

O fenômeno de habituação consiste na repetição de estímulos sensoriais, que facilita a compensação vestibular graças à plasticidade neuronal (6). O fenômeno é obtido por execução de movimentos repetitivos, que diminui a resposta vestibular e a amplitude do nistagmo. A repetição, além de promover adaptação ao movimento, estimula o órgão sensorial, criando novos automatismos responsáveis pelo equilíbrio corporal.

Dentre os métodos de reabilitação, sobressaem-se os exercícios de Cawthorne e Cooksey, criados na década de 40, por Ribeiro et al. (7). Esses exercícios consistem em movimentos cefálicos, 
Proposta de um protocolo para reabilitação vestibular em vestibulopatias periféricas

tarefas de coordenação óculo-cefálica, movimentos corporais globais e tarefas de equilíbrio. Esse método é até hoje muito utilizado e tem manifestado excelentes resultados (8), promovendo a sintomatologia e criando resposta adaptativa, por um fenômeno de neuroplasticidade (1).

Para Ayres (9), outro método, criado entre a década de 50 e 60 e que tem chamado a atenção dos profissionais da saúde, é a terapia de Integração Sensorial. O princípio fundamental desse método é conceituado por Jean Ayres, o fundador da teoria, como: "a resposta adaptável". Ocorre o desenvolvimento sensório motor, importante para o aprendizado, a integração do indivíduo com o meio de forma a desenvolver o cérebro e a capacidade da plasticidade, pelas atividades sensório-motoras (10).

Dentre as diversas práticas da integração sensorial, Ayres (9) relata a utilização do balanço, no qual o terapeuta pode interagir com o paciente por meio de brincadeiras lúdicas, estimulando o desenvolvimento sensório-motor.

Os exercícios de Cawthorne e Cooksey são bastante utilizados e apresentam ótimos resultados em pacientes com vestibulopatias periféricas. A Integração Sensorial, apesar dos bons resultados, reserva-se ao tratamento das afecções neurológicas infantis. Pela carência de maiores estudos em pacientes adultos e pelo interesse em tratar pacientes com afecções vestibulares periféricas, propõe-se criar um protocolo de exercícios em um balanço, associando os exercícios de Cawthorne e Cooksey à Integração Sensorial.

\section{Material e método}

A pesquisa foi um estudo-piloto, realizado com um indivíduo portador de disfunção vestibular periférica, atendido no Ambulatório de Fisioterapia e Terapia Ocupacional do Hospital de Clínicas da Universidade Estadual de Campinas.

Houve uma avaliação inicial realizando testes que distinguissem lesões periféricas de centrais. Lesão central foi critério de exclusão, bem como lesões ortopédicas que impedissem o indivíduo de realizar os movimentos de cervical e inclinação de tronco de maneira confortável e sem algia.

Sujeito: Paciente VBS, 47 anos, sexo feminino, com diagnóstico clínico de labirintite há 10 anos, constatado por um otorrinolaringologista, por teste calórico. Paciente relata tontura do tipo rotatória e vertigem incapacitante acompanhada de náusea. As crises aparecem de forma gradativa e esporadicamente, aumentando com mudanças de decúbito, com déficit na postura sentada e histórico de queda por causa da vertigem. Durante as crises, fazia uso de medicamento antivertiginoso e nunca realizou fisioterapia ou qualquer exercício para o tratamento da labirintite.

Instrumentos de Medidas: Escala de Equilíbrio de Berg: avalia o equilíbrio e o risco de queda, considerando o efeito do ambiente na função. Esta avalia também a habilidade do indivíduo de sentar, ficar de pé, alcançar, girar em torno de si mesmo, olhar por cima de seus ombros, ficar sobre apoio unipodal e transpor degraus. Movimento estático e antecipatório com pontuação total de 56, no qual, quanto maior a pontuação, melhor o resultado (11).

Dynamic Gait Index (DGI): avalia alterações vestibulares periféricas; o equilíbrio e o risco de queda. É composta por 8 testes, com pontuação de 0 a 24 . O índice menor ou igual a 19 indica alto risco de queda (12).

Dizziness Handicap Inventory (DHI): avaliação subjetiva composta por 25 perguntas a respeito da saúde física, emocional e da capacidade funcional do indivíduo. A somatória igual a 100 representa o pior escore, e zero o melhor possível (13).

Five Times Sit-to-Stand (FTSTS): consiste na atividade de sentar e levantar cinco vezes de uma cadeira com os membros superiores cruzados. Esta avalia também o equilibrio e força de membros inferiores (14).

Escala de Sintomas após Tratamento Fisioterapêutico (ESATF): escala que gradua a sintomatologia do paciente, após ter realizado os exercícios solicitados pelo terapeuta a cada sessão, que permite a evolução do paciente para a próxima série, se tiver realizado a série anterior com destreza e sem sintomatologia. Contém escores de 0 (nenhum sintoma permaneceu ao final do tratamento) até 4 (sintomas pioraram com as atividades terapêuticas em uma base persistente, em relação ao período pré-tratamento) (15). 
Material: Foi utilizado um balanço (Figura 1) suspenso por uma talha para suporte parcial de peso, já existente no ambulatório; 1 rolo terapêutico com $122 \mathrm{~cm}$ de comprimento, $36 \mathrm{~cm}$ de diâmetro e com peso de $8 \mathrm{~kg}$; estrutura metálica de aço carbono de $60 \mathrm{~cm}$ de largura, $4 \mathrm{~cm}$ de espessura e $150 \mathrm{~cm}$ de comprimento; correntes para sustentar o rolo de tamanho regulável de acordo com o paciente; tábua de $30 \mathrm{~cm}$ de largura, $2 \mathrm{~cm}$ de espessura e $150 \mathrm{~cm}$ de comprimento, na qual o rolo foi fixado por catracas; materiais lúdicos para as atividades terapêuticas, como bolas.

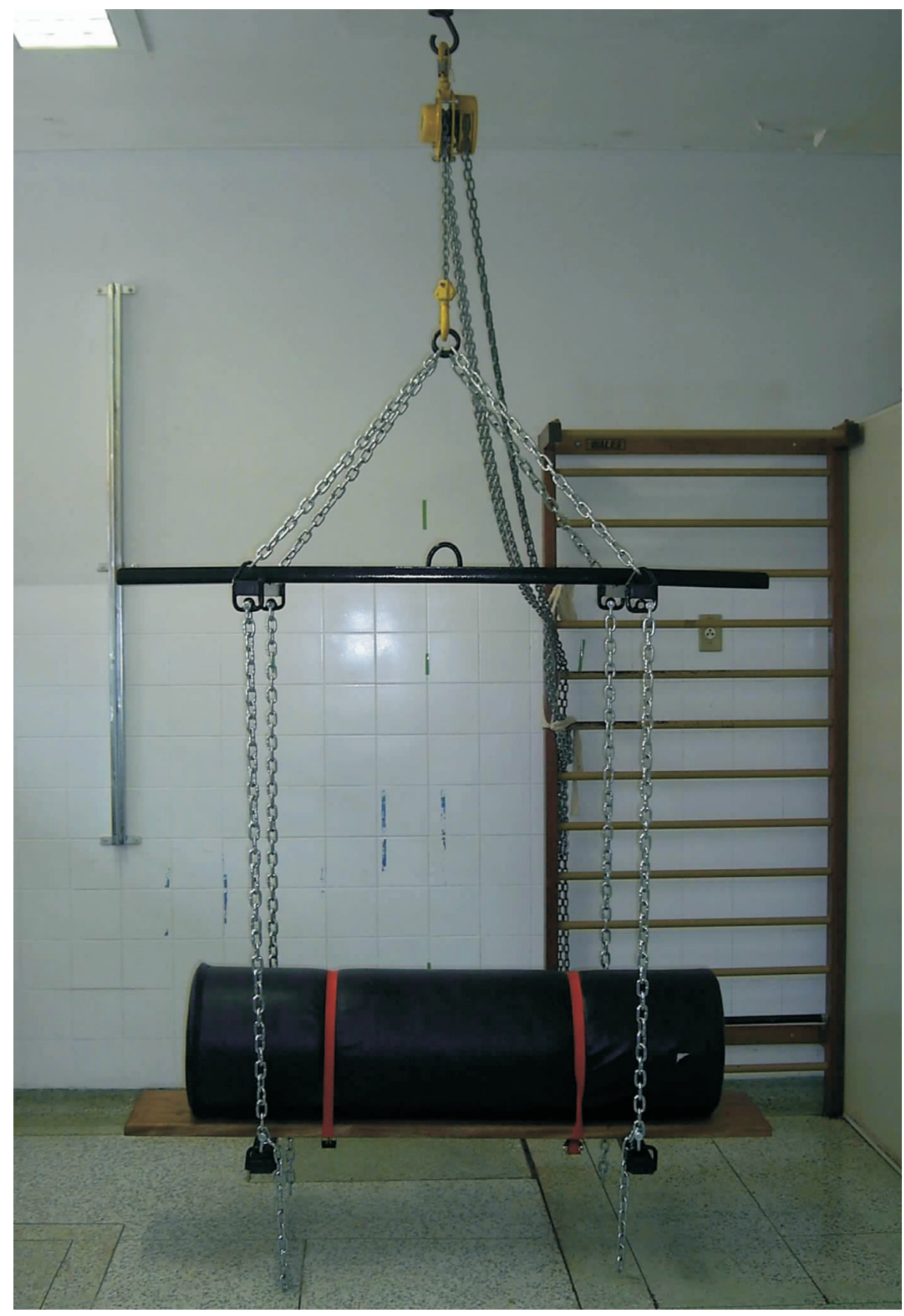

Figura 1 - Balanço suspenso por uma talha para suporte parcial de peso 
Procedimentos: A paciente incluída no estudo foi informada quanto aos procedimentos do estudo e assinou, em seguida, o termo de consentimento de forma livre e esclarecida, aprovado pelo Comitê de Ética e Pesquisa da Unicamp (n. 358/06).

O protocolo de tratamento para reabilitação vestibular proposto associou a terapia de Integração Sensorial com uso de um balanço aos exercícios de Cawthorne Cooksey (Tabela 1). Durante a aplicação do protocolo, foi pedido à paciente que não usasse medicamento antivertiginoso. $O$ protocolo foi composto por 4 tipos de atividades progressivas. Cada tipo contém de 2 a 4 tarefas, realizadas em séries de 10 repetições. A paciente somente realizava a próxima tarefa se conseguisse realizar a série anterior com destreza e sem sintomatologia, relatado pela paciente, utilizando a ESATF (15). Caso contrário, a terapia seguinte iniciava-se a partir da tarefa na qual apresentou a sintomatologia. O protocolo foi realizado em 10 sessões de 30 minutos, 2 vezes por semana.

Para a realização dos exercícios, a paciente ficava sentada no balanço, com pés apoiados, tronco alinhado, $70^{\circ}$ de flexão coxofemoral (Figura 2). O balanço é movimentado pelo terapeuta conforme o protocolo.

Tabela 1 - Protocolo de tratamento para reabilitação vestibular

I - Atividade sentada no balanço de "cavalinho" (movimentos oculares, primeiro lento e depois rápido):

a - Movimentos oculares - quando o corpo for para frente, olhos para cima, no retorno olhos para baixo (balanço anteroposterior);

b - Movimentos oculares de um lado para outro durante o balanço (balanço látero-lateral -LL-com movimentos oculares contralaterais ao balanço);

c - Concentrando o olhar em um objeto, durante o balanço (ântero-posterior -AP -e látero-lateral);

II - Atividade sentada no balanço (movimentos cefálicos, primeiro lento depois rápido - olhos abertos e olhos fechados):

a - Sentado de "cavalinho", sobre o balanço,com os pés apoiados no solo, jogar uma bola pequena, de uma mão para outra (acima do nível do horizonte), acompanhando a bola com o olhar;

b - Sentado de "cavalinho", sobre o balanço, quando o corpo for para frente olhar para o teto e quando o corpo for para trás olhar para o chão;

c - Sentado de "cavalinho", sobre o balanço, olhando para um lado e para o outro, simultaneamente ao balanço.

III - Atividades de tronco focando equilíbrio:

a - Sentado de "cavalinho", recebe a bola do terapeuta, que será jogada para diferentes lados;

b - Sentado de "cavalinho", sobre o balanço, o paciente passa a bola para o terapeuta que estará sentado atrás, de costas para o paciente (primeiro para um lado e depois para o outro).

c - Sentado de frente, inclinar o tronco para um lado, apoiando-se no cotovelo homolateral, e lateralizar a cabeça para o lado oposto;

d - Sentado de "cavalinho", durante o balanço, paciente tenta acertar objetos em um cesto ou derrubar garrafas (deslocamento AP de tronco);

e - Sentado de frente, sobre o balanço, fazer inclinação lateral do tronco contra o movimento lateral do balanço.

IV - Atividade dinâmica no balanço focando ortostatismo:

a - Levantar-se a partir da posição sentada de frente, sem utilização dos MMSS (membros superiores) e sentar novamente (primeiro olhos abertos e depois olhos fechados);

b - Levantar-se a partir da posição sentada de frente, sem utilização dos MMSS, girando o corpo a $90^{\circ}$ e sentar novamente (girar primeiro para a direita e depois para a esquerda - primeiro olhos abertos depois olhos fechados). 


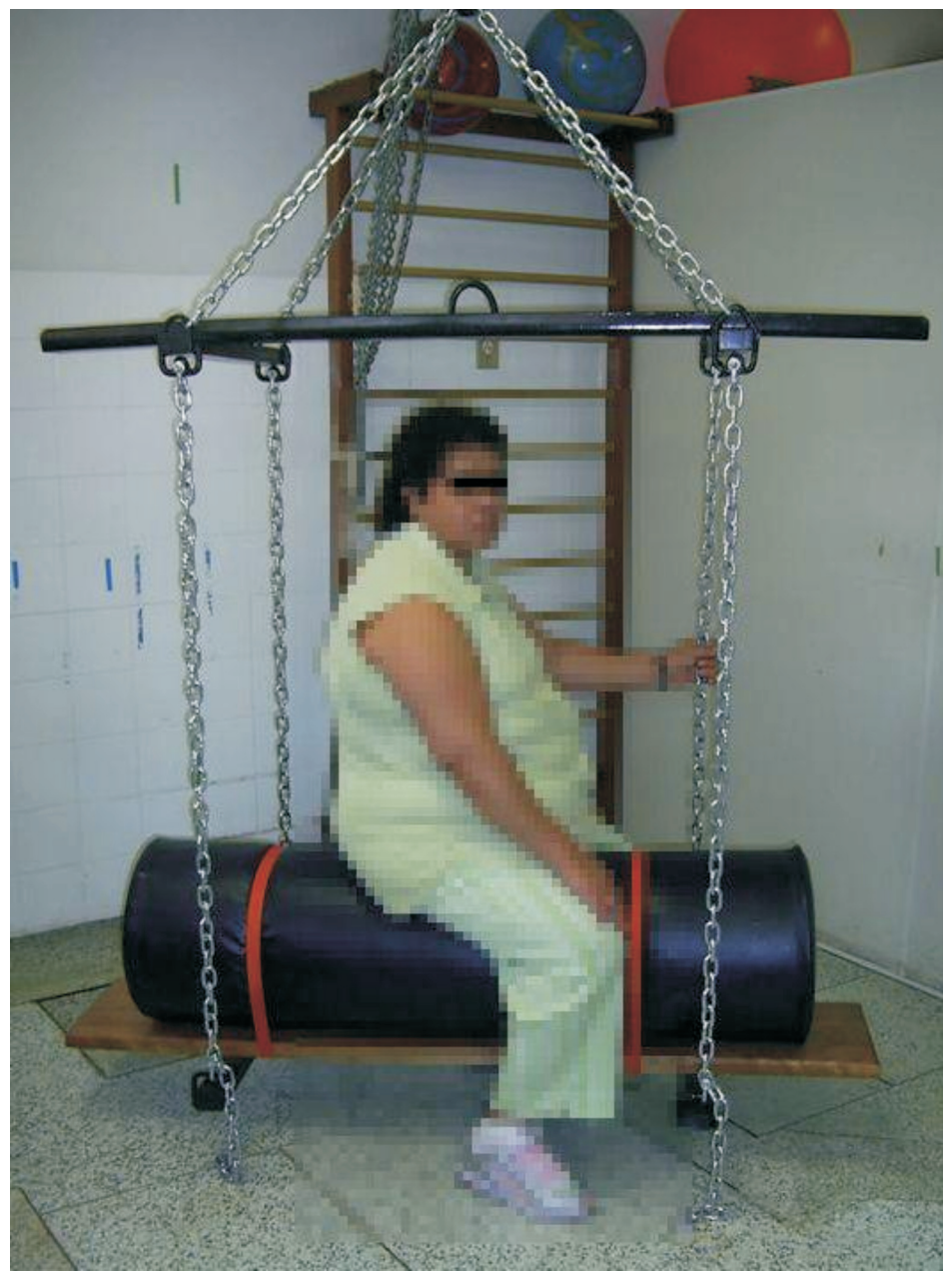

Figura 2 - Paciente sentada no balanço, com pés apoiados, tronco alinhado, $70^{\circ}$ de flexão coxofemoral

\section{Resultados}

De acordo com a escala da DGI, a paciente obteve 19 pontos antes do tratamento e 22 após, melhorando o equilíbrio e diminuindo o risco de queda.

$\mathrm{Na}$ escala de Equilíbrio de Berg, a paciente manteve pontuação de 54 antes e após o tratamento. Pela escala da DHI, a paciente obteve um total de 74 pontos na avaliação inicial e 67 pontos após a terapia, com uma melhora subjetiva da capacidade funcional.

$\mathrm{Na}$ escala de FTSTS, a paciente apresentou tempo de 15 segundos antes e 14 segundos após o tratamento. Ela diminuiu o tempo gasto para realizar a atividade de sentar e levantar cinco vezes de uma cadeira com os membros superiores cruzados (Tabela 2).

Na ESATF, que gradua a sintomatologia do paciente após os exercícios, houve oscilação da pontuação impedindo a progressão no tratamento. Dessa forma, o protocolo só pôde ser avançado três vezes.

A paciente relatou muita vertigem em alguns momentos durante os exercícios, dificultando a progressão das atividades. Além disso, ela realizou apenas 10 das 20 sessões propostas em um tempo maior que o programado, não sendo possível ocorrer a habituação. 
Tabela 2 - Pontuação das escalas mensuradas antes e após o tratamento com o balanço

\begin{tabular}{lcccc}
\hline & DGI & EEB & DHI & FTSTS (seg) \\
\hline Antes & 19 & 54 & 74 & 15 \\
Após & 22 & 54 & 67 & 14 \\
\hline
\end{tabular}

DGI: Dynamic Gait Index; EEB: Escala de Equilíbrio de Berg; DHI: Dizziness Handicap Inventory; FTSTS: Five Times Sit-to-Stand; seg: segundo.

\section{Discussão}

A reabilitação vestibular é um tratamento eficaz para pacientes com persistência da vertigem por disfunção vestibular, proporcionando acentuada melhora da qualidade de vida e no equilíbrio (3). O mecanismo de equilíbrio requer um influxo sensorial adequado do sistema visual, vestibular, proprioceptivo e somatossensorial para sua manutenção (16).

Os exercícios de Cawthorne e Cooksey têm mostrado eficácia no tratamento da reabilitação de vestibulopatias periféricas, requerendo a execução dos exercícios regularmente (17). Os exercícios são baseados no contexto de que a exposição repetitiva a um estímulo provocativo resulta em uma redução da resposta patológica a essa técnica, baseado no treinamento da habituação vestibular (18).

Silveira et al. (19) realizaram estudo comparativo entre exercícios de Cawthorne e Cooksey e exercícios seletivos de tronco em indivíduos vestibulopatas. Eles comprovaram que ambos os exercícios promoveram melhora significativa na qualidade de vida dos indivíduos, auxiliando no processo de compensação do sistema vestibular e redução dos sintomas vestibulares.

Isableu e Vuillerme (20) evidenciaram a correção da postura para indivíduos voluntários com pobre controle postural, após a técnica de Integração Sensorial. Uyangk e colaboradores (21) compararam a Integração Sensorial com excitação vestibular e terapia neuroevolutiva em crianças com Síndrome de Down e perceberam também melhora no controle motor em todas as técnicas citadas.

No protocolo proposto foi utilizada a escala de Berg para avaliar os aspectos estáticos e antecipatórios do equilíbrio (resistente à própria perturbação) (11). O escore de 54 pontos manteve-se após o tratamento. Segundo Berg (11), essa pontuação indica risco mínimo, de 3 a 4\% de queda. $\mathrm{Na}$ amplitude de 54 a 46, a alteração de um ponto é associada ao aumento de 6 a 8\% no risco de quedas, e abaixo de 36, o risco é próximo dos 100\%. Assim, a paciente conseguiu manter um escore quase máximo de normalidade, ou seja, um valor ínfimo para o risco de queda. Esse risco mínimo de queda foi confirmado pela escala da DGI, que avalia alterações vestibulares periféricas. A paciente obteve melhora na pontuação, de 19 para 22, confirmando o relato de Shumway-Cook (22), no qual o índice menor ou igual a 19 indica alto risco de queda.

Conforme os parâmetros de Jacobson e Newman (13), variação maior ou igual a 18 pontos na escala DHI (resultante da diferença de índices do pré e pós-tratamento) indica ser um bom resultado por meio da técnica de reabilitação vestibular aplicada. De acordo com esse autor, os achados da atual pesquisa não se beneficiaram com a técnica da reabilitação vestibular proposta, pois a variante foi de 7 pontos. Porém, os estudos de Hecker et al. (17) e Smith-Weelock et al. (23) relatam índice percentual de cura ou eliminação dos sintomas com valor na escala DHI inferior a 18 pontos, observando a melhora de 2 casos atendidos de pontuação 7 e 9, o que está de acordo com nosso estudo.

$\mathrm{Na}$ escala de FTSTS, a paciente diminuiu o tempo gasto para realizar a atividade solicitada, melhorando o equilíbrio e a força dos membros inferiores e, assim, a destreza de movimentos rápidos e dinâmicos.

A recuperação também pode ser prolongada ou limitada se a paciente limita os movimentos cefálicos ou se as informações visuais são minimizadas. Apesar de pacientes crônicos geralmente não 
Rogatto ARD, Pedroso L, Almeida SRM, Oberg TD.

apresentarem vertigem, limitam o movimento na tentativa de evitar a precipitação dos sintomas de desequilíbrio e náusea (24). Além disso, era importante rever a atitude motivacional da paciente, que interfere diretamente nos resultados da reabilitação vestibular (25), pois a paciente apresentava períodos de instabilidade emocional.

Quanto à ESATF, o esperado seria que o índice de sintomatologia, quantificado pela escala, diminuísse conforme a progressão no protocolo. A paciente deveria fazer 20 sessões em 2,5 meses de tratamento. Porém, pela falta de regularidade no tratamento, pois ela compareceu em apenas 10 sessões, num período de 4 meses, seus sintomas oscilaram muito nas sessões, impedindo que ocorresse a habituação.

\section{Considerações finais}

Observa-se na literatura que a reabilitação vestibular com os exercícios de Cawthorne e Cooksey e déficits sensoriais tratados por meio da Integração Sensorial, geralmente comprovam boa efetividade no tratamento, possibilitando ser promissor a utilização do protocolo proposto no tratamento de vestibulopatias.

Após o tratamento, a paciente manteve e obteve melhora no equilíbrio e na capacidade funcional e diminuiu o tempo gasto para realizar a atividade de sentar e levantar cinco vezes de uma cadeira com os membros superiores cruzados.

Sugerimos estudos adicionais utilizando o protocolo, com um número maior de pacientes e maior assiduidade, para que os exercícios possam levar a uma maior perturbação, resultando em uma habituação precoce e melhora dos sintomas.

\section{Referências}

1. Pedalini MEB, Bittar RSM. Reabilitação vestibular: uma proposta de tratamento. Pró-fono Revista de Atualização Científica. 1999;1(2):140-4.

2. Bear MF, Connors BW, Paradiso MA. Os sistemas auditivo e vestibular. In: Bear MF, Connors BW, Paradiso MA. Neurociências: desvendando o sistema nervoso. São Paulo: Artmed; 2002. p. 349-95.

3. Andersson G, Asmundson GJG, Denev J, Nilsson J, Larsen HC. A controlled trial of cognitive-behavior therapy combined with vestibular rehabilitation in the treatment of dizziness. Behav Res Ther. 2006;44(9):1265-73.

4. Ganança FF, Castro ASO, Branco FC, Natour J. Interferência da tontura na qualidade de vida de pacientes com síndrome vestibular periférica. Rev Bras Otorrinolaringol. 2004;70(1):94-101.

5. Ganança MM, Caviolla HH. Desequilíbrio e reequilíbrio. In: Ganança MM. Vertigem tem cura? São Paulo: Lemos; 1998. p. 9-13.

6. Herdman SJ, Whitney SL. Reabilitação vestibular. Barueri: Manole; 2002.

7. Ribeiro KMX, Testa JRG, Weckx LL. Labirintopatias na mulher. Rev Bras Med. [periodico online]. 2000 [capturado em 30 mar. 2008];57:1-5. Disponível em: www.cibersaude.com.br/revistas.asp?fase=r003\&id_materia=310

8. Mantello, EB, André AP, Sanches A, Colafêmina JF. A vertigem postural paroxística benigna tratada pela reabilitação vestibular - estudo de caso. Fono Atual. 2005;8(33):54-58.

9. Aires, MM. Fisiologia. Rio de Janeiro: Guanabara Koogan; 1999.

10. Ayres AJ. Sensory integration and praxis tests. Los Angeles: Western Psychological; 1989. 
11. Berg K. Measuring balance in the elderly: validation pf an instrument [dissertation]. Montreal: McGill University; 1993.

12. Shumway-Cook A, Woollacott MH. Motor control: theory and practical applications. Baltimore: Williams \& Wilkiins; 1995.

13. Jacobson GP, Newman GW. Thedevelopment of the dizziness handicap inventory. Arch Otolaryngol Head Neck Surg. 1990;152(6):386-91.

14. Lord SR, Murray SM, Chapman K, Munro B, Tiedemann A. Sit-to-stand performance depends on sensation, speed, balance and psychological status in addition to strength in older people. J Gerontol A Biol Sci Med Sci. 2002;57(8):M539-43.

15. Herdman SJ, Whitney SL. Reabilitação vestibular. Barueri: Manole; 2002.

16. Horak FB, Shupert CL, Mirka A. Components of postural dyscontrol in the elderly: a review. Neurobiol Aging. 1989;10(6):727-38.

17. Hecker HC, Haug CQ, Hernondon JW. Treatment of vertiginous patient using cawthorne's vestibular exercises. Laringoscope. 1974;84:2065-72.

18. Sawyer RN. The cervico-ocular reflex of normal human subjects in response to transient and sinusoidal trunk rotations. J Vestib Res. 1994;4(3):245-9.

19. Silveira SR, Taguchi CK, Ganança FF. Análise comparativa de duas linhas de tratamento para pacientes portadores de disfunção vestibular periférica com idade superior a sessenta anos. Acta AWHO. 2002;21(1):14-31.

20. Isableu B, Vuillerme N. Differential integration of kinaesthetic signals to postural control. Exp Brain Res. 2006;174(4):763-8.

21. Uyangk M, Bumin G, Kayghan H. Comparison of different therapy approaches in children with Down syndrome. Pediatr Int. 2003;45(1):68-73.

22. Shumway-cook A, Woollacott MH. Motor control: theory and practial applications. 2001;10:249-69.

23. Smith-Wheelock M, Shepard NT, Telian SA. Physical therapy program for vestibular rehabilitation. Am J Otol. 1991;3(12):218-25.

24. Fetter M, Zee DS. Recovery from unilateral labyrinthectomy in Rhesus monkeys. J Neurophysiol. 1988;59(2):370-93.

25. Kein RJ, Cook M, Martini D. Balance rehabilitation therapy. Laringoscope. 1992;102:1302-7.

Recebido: 29/04/2008

Received: 04/29/2008

Aprovado: 02/10/2009

Approved: 10/02/2009 